\title{
Existence of positive periodic solutions for super-linear neutral Liénard equation with a singularity of attractive type
}

\section{Yu Zhu ${ }^{1 *}$}

"Correspondence:

4040665116@qq.com

'School of Tencent Cloud Big Data,

Ma'anshan University, Maanshan, China

\section{Springer}

\begin{abstract}
In this paper, the existence of positive periodic solutions is studied for super-linear neutral Liénard equation with a singularity of attractive type

$$
(x(t)-c x(t-\sigma))^{\prime \prime}+f(x(t)) x^{\prime}(t)-\varphi(t) x^{\mu}(t)+\frac{\alpha(t)}{x^{\gamma}(t)}=e(t),
$$

where $f:(0,+\infty) \rightarrow R, \varphi(t)>0$ and $\alpha(t)>0$ are continuous functions with $T$-periodicity in the $t$ variable, $c, \gamma$ are constants with $|c|<1, \gamma \geq 1$. Many authors obtained the existence of periodic solutions under the condition $0<\mu \leq 1$, and we extend the result to $\mu>1$ by using Mawhin's continuation theorem as well as the techniques of a priori estimates. At last, an example is given to show applications of the theorem.
\end{abstract}

Keywords: Periodic solution; Neutral equation; Mawhin's continuation theorem; Attractive singularity

\section{Introduction}

The second order differential equations with singularities have a wide range of applications in many subjects, such as physics, engineering, mechanics, and so on (see [1-5]). After the pioneering paper [6] came out, many scholars put their attention to the periodic problems of second order singular differential equations without friction term (see [7-13] and the references therein). Beginning with the paper of Habets-Sanchez in [14], the interest in the problem of periodic solution for second order singular differential equations with friction term has increased [15-21]. Hakl, Torres, and Zamora considered the periodic problem for the singular equation of attractive type

$$
x^{\prime \prime}(t)+f(x(t)) x^{\prime}(t)-\varphi(t) x^{\mu}(t)+\frac{g_{1}}{x^{\gamma}(t)}=0,
$$

where $\mu \in(0,1]$ is a constant, $\varphi$ is a $T$-periodic function with $\varphi \in L^{1}([0, T], R), f \in$ $C((0,+\infty), R)$. By using Schauder's fixed point theorem, as well as upper and lower functions method, they obtained the following result (Theorem 3.16, [22]).

(c) The Author(s) 2020. This article is licensed under a Creative Commons Attribution 4.0 International License, which permits use, sharing, adaptation, distribution and reproduction in any medium or format, as long as you give appropriate credit to the original author(s) and the source, provide a link to the Creative Commons licence, and indicate if changes were made. The images or other third party material in this article are included in the article's Creative Commons licence, unless indicated otherwise in a credit line to the material. If material is not included in the article's Creative Commons licence and your intended use is not permitted by statutory regulation or exceeds the permitted use, you will need to obtain permission directly from the copyright holder. To view a copy of this licence, visit http://creativecommons.org/licenses/by/4.0/ 
Theorem 1.1 Let $\mu=1$ and $g_{1}>0$. If $\int_{0}^{T} \varphi(s) d s>0$ and

$$
\frac{T}{4} \int_{0}^{T} \varphi_{+}(s) d s \int_{0}^{T} \varphi_{-}(s) d s<\int_{0}^{T} \varphi_{+}(s) d s-\int_{0}^{T} \varphi_{-}(s) d s,
$$

where $\varphi_{+}(t)=\max \{\varphi(t), 0\}$ and $\varphi_{-}(t)=\min \{\varphi(t), 0\}$, then there exists at least one positive periodic solution to Eq. (1.1). However, the study of periodic solutions for delay functional differential equation with a singularity is relatively infrequent [23-25]. For example, Wang in [23] studied the problem of periodic solutions for the singular delay Liénard equation of repulsive type

$$
x^{\prime \prime}(t)+f(x(t)) x^{\prime}(t)+\varphi(t) x^{\mu}(t-\tau)-\frac{1}{x^{\gamma}(t-\tau)}=e(t),
$$

and the periodic problem for neutral Liénard equation of repulsive type

$$
(x(t)-c x(t-\sigma))^{\prime \prime}+f(x(t)) x^{\prime}(t)+\varphi(t) x^{\mu}(t-\tau)-\frac{\alpha(t)}{x^{\gamma}(t)}=h(t)
$$

has been investigated in [24] and [25], where $\varphi(t)$ and $e(t)$ are T-periodic with $\varphi, e \in$ $L^{1}[0, T], \gamma$ and $\mu$ are positive constants. We notice that the degree associated with the term $\varphi(t) x^{\mu}$ is required $\mu \in(0,1)$.

Motivated by these, in this paper, we continue to study the periodic problem for neutral Liénard equation with a singularity of attractive type

$$
(x(t)-c x(t-\sigma))^{\prime \prime}+f(x(t)) x^{\prime}(t)-\varphi(t) x^{\mu}(t)+\frac{\alpha(t)}{x^{\gamma}(t)}=e(t),
$$

where $f, \varphi$ are the same as the ones of Eq. (1.4). $e$ is a $T$-periodic function with $e \in$ $L^{1}([0, T], R)$ and $\int_{0}^{T} e(s) d s=0$. By means of a continuation theorem of the coincidence degree principle developed by Manásevich and Mawhin, as well as the techniques of a priori estimates, some new results on the existence of positive periodic solutions are obtained. The interesting point in this paper is that the constant $\mu$ is allowed $\mu \in(0,2]$. It is easy to see that if $\mu \in(1,2]$, the restoring force term $\varphi(t) x^{\mu}$ is super-linear with respect to $x$.

The rest of this paper is organized as follows. In Sect. 2, we state some necessary definitions and lemmas. In Sect. 3, we prove the main result. At last, we give an example of an application in Sect. 4.

\section{Essential definitions and lemmas}

In this section, we define $X=C_{T}^{1}=\left\{x \in C^{1}(R, R): x(t+T)=x(t), \forall t \in[0, T]\right\}$ with the norm $\|x\|_{C_{T}^{1}}=\max \left\{\|x\|_{\infty},\left\|x^{\prime}\right\|_{\infty}\right\}$ and $Y=C_{T}=\{x \in C(R, R): x(t+T)=x(t), \forall t \in[0, T]\}$ with the norm $\|x\|_{\infty}=\max _{t \in[0, T]}|x(t)|$. For $y \in C_{T}, y_{m}$ is denoted by $\min _{t \in[0, T]} y(t)$. Clearly, $C_{T}$ and $C_{T}^{1}$ are Banach spaces. Denote the operator L as follows:

$$
L x=(A x)^{\prime \prime}, \quad L:=D(L) \subset C_{T}^{1} \rightarrow C_{T},
$$


where $A: C_{T} \rightarrow C_{T},(A x)(t)=x(t)-c x(t-\sigma)$, and $D(L)=\left\{x \in C_{T}^{1}: A x \in C^{2}(R, R)\right\}$, and

$$
\begin{aligned}
& (A x)^{\prime \prime}(t)+f(x(t)) x^{\prime}(t)-\varphi(t) x^{\mu}(t)+\frac{\alpha(t)}{x^{\gamma}(t)}=e(t), \\
& (N x)(t)=-f(x(t)) x^{\prime}(t)+\varphi(t) x^{\mu}(t)-\frac{\alpha(t)}{x^{\gamma}(t)}+e(t), \quad N: \Delta \rightarrow C_{T},
\end{aligned}
$$

where $\Delta=\left\{x \in C_{T}^{1}: x(t)>0, \forall t \in[0, T]\right\}$. Then Eq. (1.5) (or Eq. (2.1)) can be rewritten as $L x=N x$.

Lemma 2.1 ([27]) If $|c|<1$, then $A$ has continuous inverse on $C_{T}$ and

(1) $\left\|A^{-1} x\right\|_{\infty} \leq \frac{\|x\|_{\infty}}{|1-| c||}$ for all $x \in C_{T}$;

(2) $\int_{0}^{T}\left|\left(A^{-1} f\right)(t)\right| d t \leq \frac{1}{|1-| c||} \int_{0}^{T}|f(t)| d t$ for all $f \in C_{T}$;

(3) $\int_{0}^{T}\left|\left(A^{-1} f\right)\right|^{2}(t) \mid d t \leq \frac{1}{(1-|c|)^{2}} \int_{0}^{T} f^{2}(t) d t$ for all $f \in C_{T}$.

Lemma 2.2 ([23]) Let $x(t)$ be a continuously differentiable T-periodic function. Then there is a point $\xi \in[0, T]$

$$
\left(\int_{0}^{T} x^{2}(t) d t\right)^{\frac{1}{2}} \leq \frac{T^{\frac{1}{2}}}{2}\left(\int_{0}^{T} x^{\prime 2}(t) d t\right)^{\frac{1}{2}}+x(\xi)
$$

Lemma 2.3 ([22]) Let $u(t):[0, \omega] \rightarrow R$ be an arbitrary absolutely continuous function with $u(0)=u(\omega)$. Then the inequality

$$
\left(\max _{t \in[0, \omega]} u(t)-\min _{t \in[0, \omega]} u(t)\right)^{2} \leq \frac{\omega}{4} \int_{4}^{\omega}\left|u^{\prime}(s)\right|^{2} d s
$$

holds.

Lemma 2.4 ([26]) Let $X$ and $Y$ be two real Banach spaces, let $\Omega$ be an open and bounded set of $X$, and let $L: D(L) \subset X \rightarrow Y$ be a Fredholm operator of index zero, and the operator $N: \bar{\Omega} \subset X \rightarrow Y$ is said to be L-compact in $\bar{\Omega}$. In addition, if the following conditions hold:

(1) $L x \neq \lambda N x$ for all $(x, \lambda) \in \partial \Omega \times(0,1)$;

(2) $Q N x \neq 0$ for all $x \in \operatorname{ker} L \bigcap \partial \Omega$;

(3) $\operatorname{deg}\{J Q N, \Omega \bigcap \operatorname{ker} L, 0\}$, where $J: \operatorname{Im} Q \rightarrow \operatorname{ker} L$ is a homeomorphism.

Then $L x=N x$ has at least one solution in $D(L) \bigcap \bar{\Omega}$.

Remark 2.5 If $\bar{\varphi}>0, \bar{e}=0$, then there are constants $C_{1}$ and $C_{2}$ with $0<C_{1}<C_{2}$ such that

$$
\bar{\varphi} x^{\mu}-\frac{1}{x^{\gamma}}>0, \quad \forall x \in\left(0, C_{1}\right)
$$

and

$$
\bar{\varphi} x^{\mu}-\frac{1}{x^{\gamma}}<0, \quad \forall x \in\left(C_{2},+\infty\right)
$$

Now, we list the following assumptions, which will be used in Sect. 3 for investigating the existence of positive $T$-periodic solution to Eq. (2.1): 
(H1) $1-|c|>0$,

(H2) $1-\frac{\sqrt{T N_{2}}}{2}>0$, where $N_{2}=\frac{T \bar{\varphi}\left(\frac{1}{|\varphi| \infty}\right)^{\frac{1}{\mu+\gamma}}}{1-|c|}$.

\section{Main results}

Now, we embed Eq. (1.3) into the following equations family with a parameter $\lambda \in(0,1]$ :

$$
(x(t)-c x(t-\sigma))^{\prime \prime}+\lambda f(x(t)) x^{\prime}(t)-\lambda \varphi(t) x^{\mu}(t)+\lambda \frac{\alpha(t)}{x^{\gamma}(t)}=\lambda e(t) .
$$

Let

$$
D=\left\{x \in C_{T}^{1}: L x=\lambda N x, \lambda \in(0,1]\right\} .
$$

Theorem 3.1 Assume $\bar{\varphi}>0, \alpha(t)>0$, and $\bar{e}=0$, then there are two constants $\tau_{0}, \tau_{1} \in[0, T]$ for each $u \in D$ such that

$$
u\left(\tau_{0}\right) \leq \max \left\{1,\left(\frac{\bar{\alpha}}{\bar{\varphi}}\right)^{\frac{1}{\mu}}\right\}:=A_{0}
$$

and

$$
u\left(\tau_{1}\right) \geq \min \left\{1,\left(\frac{\bar{\alpha}}{\bar{\varphi}}\right)^{\frac{1}{\mu}}\right\}:=A_{1}
$$

Proof Let $u \in D$, then

$$
(A u)^{\prime \prime}(t)+\lambda f(u(t)) u^{\prime}(t)-\lambda \varphi(t) u^{\mu}(t)+\frac{\lambda \alpha(t)}{u^{\gamma}(t)}=\lambda e(t) .
$$

Integrating both sides of Eq. (3.5) over the interval $[0, T]$, we obtain that

$$
\int_{0}^{T} \varphi(t) u^{\mu}(t) d t=\int_{0}^{T} \frac{\alpha(t)}{u^{\gamma}(t)} d t
$$

If $u(t)>1$, combine with the mean value theorem of integrals and $\varphi(t)>0$, then

$$
u^{\mu}(\tau) T \bar{\varphi}=\int_{0}^{T} \varphi(t) u^{\mu}(t) d t=\frac{T \bar{\alpha}}{u^{\gamma}(\xi)}<T
$$

which yields

$$
u(\tau)<\left(\frac{\bar{\alpha}}{\bar{\varphi}}\right)^{\frac{1}{\mu}}
$$

So there exists a point $\tau_{0} \in[0, T]$ such that

$$
u\left(\tau_{0}\right) \leq \max \left\{1,\left(\frac{\bar{\alpha}}{\bar{\varphi}}\right)^{\frac{1}{\mu}}\right\}:=A_{0} .
$$


On the other hand, when $\varphi(t)>0$ for every $\mu \in D$, there always exists a point $\tau_{1} \in[0, T]$ such that

$$
u\left(\tau_{1}\right) \geq \min \left\{1,\left(\frac{\bar{\alpha}}{\bar{\varphi}}\right)^{\frac{1}{\mu}}\right\}:=A_{1}
$$

Theorem 3.2 Suppose that assumptions of $\left[\mathrm{H}_{1}\right]$ and $\left[\mathrm{H}_{2}\right]$ hold, and $\bar{\varphi}>0, \bar{e}=0$, then Eq. (2.1) has at least one positive T-periodic solution.

Proof Suppose $u \in D$, then Eq. (3.5) holds. By multiplying both sides of Eq. (3.5) by $u(t)$ and integrating it over the interval $[0, T]$, we get

$$
\int_{0}^{T}(A u)^{\prime \prime}(t) u(t) d t=\lambda \int_{0}^{T} \varphi(t) u^{\mu}(t) u(t) d t-\lambda \int_{0}^{T} \frac{u(t) \alpha(t)}{u^{\gamma}(t)} d t+\lambda \int_{0}^{T} e(t) u(t) d t .
$$

Since

$$
\int_{0}^{T}(A u)^{\prime \prime}(t) u(t) d t=-\int_{0}^{T}\left|u^{\prime}(t)\right|^{2} d t+c \int_{0}^{T} u^{\prime}(t) u^{\prime}(t-\sigma) d t
$$

it is easy to verify that

$$
\begin{aligned}
\int_{0}^{T}\left|u^{\prime}(t)\right|^{2} d t= & c \int_{0}^{T} u^{\prime}(t) u^{\prime}(t-\sigma) d t \\
& +\lambda \int_{0}^{T}\left(\frac{\alpha(t)}{u^{\gamma}(t)}-\varphi(t) u^{\mu}(t)\right) u(t) d t-\lambda \int_{0}^{T} e(t) u(t) d t .
\end{aligned}
$$

Let $A_{2}=\left(\frac{\alpha_{m}}{|\varphi|_{\infty}}\right)^{\frac{1}{\mu+\gamma}}$ and $A_{3}=\left(\frac{|\alpha|_{\infty}}{\varphi_{m}}\right)^{\frac{1}{\mu+\gamma}}$. Set $E_{1}=\left\{t \in[0, T]: 0<u(t)<A_{2}\right\}, E_{2}=\{t \in$ $\left.[0, T]: A_{2} \leq u(t) \leq A_{3}\right\}, E_{3}=\left\{t \in[0, T]: u(t)>A_{3}\right\}$, and $E_{1} \bigcup E_{2} \bigcup E_{3}=[0, T]$. We can obtain

$$
\begin{aligned}
\int_{0}^{T}\left|u^{\prime}(t)\right|^{2} d t= & c \int_{0}^{T} u^{\prime}(t) u^{\prime}(t-\sigma) d t+\lambda \int_{E_{1}}\left(\frac{\alpha(t)}{u^{\gamma}(t)}-\varphi(t) u^{\mu}(t)\right) u(t) d t \\
& +\lambda \int_{E_{2}}\left(\frac{\alpha(t)}{u^{\gamma}(t)}-\varphi(t) u^{\mu}(t)\right) u(t) d t+\lambda \int_{E_{3}}\left(\frac{\alpha(t)}{u^{\gamma}(t)}\right. \\
& \left.-\varphi(t) u^{\mu}(t)\right) u(t) d t-\lambda \int_{0}^{T} e(t) u(t) d t \\
\leq & |c| \int_{0}^{T}\left|u^{\prime}(t)\right|^{2} d t+\left|\int_{E_{1}}\left(\frac{\alpha(t)}{u^{\gamma}(t)}-\varphi(t) u^{\mu}(t)\right) u(t) d t\right| \\
& +\int_{E_{2}}\left|\left(\frac{\alpha(t)}{u^{\gamma}(t)}-\varphi(t) u^{\mu}(t)\right) u(t)\right| d t+T\|u\|_{\infty} \overline{e_{-}} .
\end{aligned}
$$

It follows that

$$
\begin{aligned}
(1-|c|) \int_{0}^{T}\left|u^{\prime}(t)\right|^{2} d t \leq & A_{2} \int_{E_{1}}\left(\frac{\alpha(t)}{u^{\gamma}(t)}-\varphi(t) u^{\mu}(t)\right) d t \\
& +A_{3} N_{0}+T\|u\|_{\infty} \overline{e_{-}}
\end{aligned}
$$


where $N_{0}=T \max _{A_{2}<x<A_{3}, t \in[0, T]}\left\{\left|\frac{\alpha(t)}{x^{\gamma}}-\varphi(t) x^{\mu}\right|\right\}$. On the other hand,

$$
\left.\left(\int_{E_{1}}+\int_{E_{2}}+\int_{E_{3}}\right)\left(\frac{\alpha(t)}{u^{\gamma}(t)}-\varphi(t) u^{\mu}(t)\right) d t\right)=0
$$

Combining it with Eq. (3.9) we get that

$$
\begin{aligned}
(1-|c|) & \int_{0}^{T}\left|u^{\prime}(t)\right|^{2} d t \\
\leq & A_{2}\left(-\int_{E_{2}}\left(\frac{\alpha(t)}{u^{\gamma}(t)}-\varphi(t) u^{\mu}(t)\right) d t-\int_{E_{3}}\left(\frac{\alpha(t)}{u^{\gamma}(t)}-\varphi(t) u^{\mu}(t)\right) d t\right) \\
& +A_{3} N_{0}+T\|u\|_{\infty} \overline{e_{-}} \\
\leq & A_{2}\left|\int_{E_{2}}\left(\frac{\alpha(t)}{u^{\gamma}(t)}-\varphi(t) u^{\mu}(t)\right) d t\right|+A_{2}\left|\int_{E_{3}}\left(\frac{\alpha(t)}{u^{\gamma}(t)}-\varphi(t) u^{\mu}(t)\right) d t\right| \\
& +A_{3} N_{0}+T\|u\|_{\infty} \overline{e_{-}} \\
\leq & A_{2} N_{0}+A_{2} \int_{E_{3}} \frac{\alpha(t)}{u^{\gamma}(t)} d t+A_{2} \int_{E_{3}} \varphi(t) u^{\mu}(t) d t \\
& +A_{3} N_{0}+T\|u\|_{\infty} \overline{e_{-}} \\
\leq & \left(A_{2}+A_{3}\right) N_{0}+\frac{A_{2} T \bar{\alpha}}{A_{3}^{\gamma}}+A_{2} T \bar{\varphi}\|u\|_{\infty}^{\mu}+T\|u\|_{\infty} \overline{e_{-}}
\end{aligned}
$$

From the condition, we see that

$$
\int_{0}^{T}\left|u^{\prime}(t)\right|^{2} d t \leq \frac{\left[\left(A_{2}+A_{3}\right) N_{0}+\frac{A_{2} T \bar{\alpha}}{A_{3}}\right]}{1-|c|}+\frac{A_{2} T \bar{\varphi}\|u\|_{\infty}^{\mu}}{1-|c|}+\frac{T\|u\|_{\infty} \overline{e_{-}}}{1-|c|} .
$$

Let $N_{1}=\frac{\left[\left(A_{2}+A_{3}\right) N_{0}+\frac{A_{2} T \bar{\alpha}}{A_{3}}\right]}{1-|c|}, N_{2}=\frac{A_{2} T \bar{\varphi}}{1-|c|}$, and $N_{3}=\frac{T \overline{e_{-}}}{1-|c|}$, we have

$$
\int_{0}^{T}\left|u^{\prime}(t)\right|^{2} d t \leq N_{1}+N_{2}\|u\|_{\infty}^{\mu}+N_{3}\|u\|_{\infty}
$$

By using Lemma 2.2, we obtain that

$$
\begin{aligned}
\|u\|_{\infty} & \leq A_{0}+\frac{\sqrt{T}}{2}\left(\int_{0}^{T}\left|u^{\prime}(t)\right|^{2}\right)^{\frac{1}{2}} \\
& \leq A_{0}+\frac{\sqrt{T}}{2}\left(N_{1}+N_{2}\|u\|_{\infty}^{\mu}+N_{3}\|u\|_{\infty}\right)^{\frac{1}{2}} \\
& \leq A_{0}+\frac{\sqrt{T}}{2} N_{1}^{\frac{1}{2}}+\frac{\sqrt{T}}{2} N_{2}^{\frac{1}{2}}\|u\|_{\infty}^{\frac{\mu}{2}}+\frac{\sqrt{T}}{2} N_{3}^{\frac{1}{2}}\|u\|_{\infty}^{\frac{1}{2}} .
\end{aligned}
$$

Now, we begin to estimate a priori upper bounds of $u(t)$. In order to do this, we divide the estimation into two cases.

Case 1: $0<\mu<2$. From Eq. (3.12), it is easy to see that there exists a constant $M_{1}>0$ such that

$$
\|u\|_{\infty}<M_{1}
$$


Case 2: $\mu=2$. For this case, Eq. (3.12) can be rewritten as

$$
\left(1-\frac{\sqrt{T}}{2} N_{2}^{\frac{1}{2}}\right)\|u\|_{\infty} \leq A_{0}+\frac{\sqrt{T}}{2} N_{1}^{\frac{1}{2}}+\frac{\sqrt{T}}{2} N_{3}^{\frac{1}{2}}\|u\|_{\infty}^{\frac{1}{2}}
$$

which together with assumption $\left[H_{2}\right]$ yields that there exists a constant $M_{2}>0$ such that

$$
\|u\|_{\infty}<M_{2}
$$

Thus, in either case 1 or case 2 , we have

$$
\|u\|_{\infty}<\max \left\{M_{1}, M_{2}\right\}:=M_{3} .
$$

Substituting Eq. (3.13) into Eq. (3.11), we have that there exists a constant $M_{4}>0$ such that $\int_{0}^{T}\left|u^{\prime}(t)\right|^{2} d t<M_{4}$. Since $A u \in C_{T}^{1}$, there is $t_{0} \in[0, T]$ such that $(A u)^{\prime}\left(t_{0}\right)=0$. From Eq. (3.5), we get

$$
\begin{aligned}
&\left|(A u)^{\prime}(t)\right|= \lambda\left|\int_{t_{0}}^{t}\left[-f(u(t)) u^{\prime}(t)+\varphi(t) u^{\mu}(t)-\frac{\alpha(t)}{u^{\gamma}(t)}+e(t)\right] d t\right| \\
& \leq \int_{0}^{T}|f(u(t))|\left|u^{\prime}(t)\right| d t+\int_{0}^{T}\left|\varphi(t) u^{\mu}(t)\right| d t \\
&+\int_{0}^{T}\left|\frac{\alpha(t)}{u^{\gamma}(t)}\right| d t+\int_{0}^{T}|e(t)| d t \\
& \leq|f|_{M_{3}} T^{\frac{1}{2}}\left(\int_{0}^{T}\left|u^{\prime}(t)\right|^{2}\right)^{\frac{1}{2}}+2 T \overline{|\varphi|} M_{3}^{\mu}+T \overline{|e|} \\
& \leq|f|_{M_{3}} T^{\frac{1}{2}}\left(M_{4}\right)^{\frac{1}{2}}+2 T \overline{|\varphi|} M_{3}^{\mu}+T \overline{|e|}:=M_{5},
\end{aligned}
$$

where $|f|_{M_{3}}:=\max _{0 \leq x \leq M_{3}}|f(x)|$. By using Lemma 2.1, we get the inequality

$$
\left|u^{\prime}\right|_{\infty}<\left|A^{-1} A u^{\prime}\right|_{\infty} \leq \frac{\left|A u^{\prime}\right|_{\infty}}{1-|c|} \leq \frac{M_{5}}{1-|c|}:=M_{6} .
$$

In what follows, we will show the estimation

$$
\min _{t \in[0, T]} u(t)>\gamma_{0}, \quad \text { uniformly for all } u \in D
$$

where $\gamma_{0}>0$ is a constant, $D$ is determined in Eq. (3.2).

Let $\tau_{1}$ be determined in Eq. (3.8). Multiplying both sides of Eq. (3.5) by $u^{\prime}(t)$ and integrating it over the interval $\left[t, \tau_{1}\right]$, we obtain that

$$
\begin{aligned}
& \int_{t}^{\tau_{1}}\left[(A u)^{\prime \prime}(s) u^{\prime}(s)+\lambda f(u(s))\left|u^{\prime}(s)\right|^{2}-\lambda \varphi(s) u^{\mu}(s) u^{\prime}(s)+\frac{\lambda u^{\prime}(s) \alpha(s)}{u^{\gamma}(s)}\right] d s \\
& \quad=\lambda \int_{t}^{\tau_{1}} e(s) u^{\prime}(s) d s .
\end{aligned}
$$


Because of $\int_{t}^{\tau_{1}} \frac{u^{\prime}(s) \alpha(s)}{u^{\gamma(s)}} d s=\int_{t}^{\tau_{1}} \frac{\alpha(s) d u(s)}{u^{\gamma(s)}}=\int_{u(t)}^{u\left(\tau_{1}\right)} \frac{\alpha(s) d v}{v^{\gamma}}$, we can get from Eq. (3.13) and Eq. (3.14) that

$$
\begin{aligned}
\lambda\left|\int_{u(t)}^{u\left(\tau_{1}\right)} \frac{\alpha(s) d v}{v^{\gamma}}\right| & \\
\leq & \int_{t}^{\tau_{1}}\left|(A u)^{\prime \prime}(s)\right|\left|u^{\prime}(s)\right| d s+\lambda \int_{t}^{\tau_{1}}|f(u(s))|\left|u^{\prime}(s)\right|^{2} d s \\
& +\lambda \int_{t}^{\tau_{1}}|\varphi(s)|\left|u^{\mu}(s)\right|\left|u^{\prime}(s)\right| d s+\lambda \int_{t}^{\tau_{1}}|e(s)|\left|u^{\prime}(s)\right| d s \\
\leq & \left|u^{\prime}\right|_{\infty} \int_{0}^{T}\left|(A u)^{\prime \prime}(s)\right| d s+\lambda\left|u^{\prime}\right|_{\infty}^{2} \int_{0}^{T}|f(u(s))| d s \\
& +\lambda\left|u^{\prime}\right|_{\infty}|u|_{\infty}^{\mu} \int_{0}^{T}|\varphi(s)| d s+\lambda\left|u^{\prime}\right|_{\infty} \int_{0}^{T}|e(s)| d s \\
\leq & M_{6} \int_{0}^{T}\left|(A u)^{\prime \prime}(s)\right| d s+\lambda M_{6}^{2}|f|_{M_{3}}+\lambda M_{6} M_{3}^{\mu} T \overline{|\varphi|}+\lambda M_{6} T \overline{|e|} .
\end{aligned}
$$

Furthermore, integrating Eq. (3.5) over the interval $[0, T]$,

i.e.,

$$
\begin{aligned}
\int_{0}^{T}(A u)^{\prime \prime}(t) d t= & -\lambda \int_{0}^{T} f(u(t)) u^{\prime}(t) d t+\lambda \int_{0}^{T} \varphi(t) u^{\mu}(t) d t \\
& -\lambda \int_{0}^{T} \frac{\alpha(t)}{u^{\gamma}(t)} d t+\lambda \int_{0}^{T} e(t) d t
\end{aligned}
$$

it is clear that

$$
\begin{aligned}
\int_{0}^{T}\left|(A u)^{\prime \prime}(t)\right| d t \leq & \lambda \int_{0}^{T}\left|f(u(t)) u^{\prime}(t)\right| d t+\lambda \int_{0}^{T}|\varphi(t)|\left|u^{\mu}(t)\right| d t \\
& +\lambda \int_{0}^{T}|e(t)| d t \\
\leq & \lambda\left(|f|_{M_{3}} M_{6}+2 T \overline{|\varphi|} M_{3}^{\mu}+2 T \overline{|e|}\right) .
\end{aligned}
$$

Now, substituting Eq. (3.17) into Eq. (3.16), we have

$$
\begin{aligned}
\left|\int_{u(t)}^{u\left(\tau_{1}\right)} \frac{\alpha(s) d v}{v^{\gamma}}\right| \leq & M_{6}\left[|f|_{M_{3}} M_{6}+2 T \overline{|\varphi|} M_{3}^{\mu}+2 T \overline{|e|}\right] \\
& +M_{6}^{2}|f|_{M_{3}}+M_{6} M_{3}^{\mu} T \overline{|\varphi|}+M_{6} T \overline{|e|}:=M_{7}
\end{aligned}
$$

and so

$$
\left|\int_{u(t)}^{u\left(\tau_{1}\right)} \frac{\alpha(s) d v}{v^{\gamma}}\right| \leq M_{7} \quad \forall t \in\left[\tau_{1}, \tau_{1}+T\right] .
$$

On the other hand, when $\gamma \geq 1$, we have $\int_{0}^{A_{1}} \frac{\alpha_{m}}{v^{\gamma}} d v=+\infty$, then there exists $\gamma_{0} \in\left(0, A_{1}\right)$ such that

$$
\left|\int_{\epsilon}^{A_{1}} \frac{\alpha_{m} d \nu}{v^{\gamma}}\right|>M_{7} \quad \forall \epsilon \in\left(0, \gamma_{0}\right] .
$$


And if $t^{*} \in\left[\tau_{1}, \tau_{1}+T\right]$ such that $u\left(t^{*}\right) \leq \gamma_{0}$, from Eq. (3.19) we see that

$$
\left|\int_{u\left(t^{*}\right)}^{u\left(\tau_{1}\right)} \frac{\alpha(s) d v}{\nu^{\gamma}}\right|>\int_{u\left(t^{*}\right)}^{A_{1}} \frac{\alpha_{m} d \nu}{\nu^{\gamma}}>M_{7}
$$

which contradicts Eq. (3.18). This contradiction verifies Eq. (3.15). From Eq. (3.13), Eq. (3.14), and Eq. (3.15), as well as the inequality in Remark 2.5, we can verify all the conditions of Lemma 2.4. Thus, by using Lemma 2.4, we see that Eq. (3.5) has at least one positive $T$-periodic solution.

\section{Example}

In this section, we present an example to demonstrate the main results.

Example 4.1 Consider the following equation:

$$
(u(t)-0.1 u(t-\sigma))^{\prime \prime}+f(u(t)) u^{\prime}(t)-a(1+\cos t) u^{\frac{3}{2}}(t)+\frac{1}{u^{\frac{3}{2}}}=\sin t
$$

Corresponding to Eq. (3.5), in (4.1), $c=0.1, \varphi(t)=a(1+\cos t), a>0, e(t)=\sin t$, and $T=2 \pi$. Obviously, $\bar{\varphi}=a$ and $\bar{e}=0$ for all $t \in[0, T]$ with $|\varphi|_{\infty}=2 a, A_{2}=\left(\frac{1}{2 a}\right)^{\frac{1}{3}}$, and $N_{2}=\frac{\left(\frac{1}{2 a}\right)^{\frac{1}{3}} 2 \pi a}{1-0.1}=$ $\frac{\frac{1}{\sqrt[3]{2}} 2 \pi a^{\frac{2}{3}}}{0.9}$. Since $(4.1)$ satisfies $(\mathrm{H} 2)$

i.e.,

$$
1-\frac{\sqrt{T} N_{2}}{2}=1-\frac{\sqrt{2 \pi}\left(\frac{\frac{1}{\sqrt[3]{2}} 2 \pi a^{\frac{2}{3}}}{0.9}\right)^{\frac{1}{2}}}{2}>0
$$

we get that $a<\frac{\sqrt{2}(0.9)^{\frac{3}{2}}}{\pi^{3}}$. Thus, by using Theorem 3.1 and Theorem 3.2, when $a<\frac{\sqrt{2}(0.9)^{\frac{3}{2}}}{\pi^{3}}$, Eq. (4.1) has at least one positive $2 \pi$-periodic solution.

Remark 4.2 From the above example, we see that the degree $\mu$ associated with the restoring force term $\varphi(t) x^{\mu}$ is $\mu=\frac{3}{2}$, which is different from the corresponding ones of $\mu \in(0,1]$ in [23-25]. Furthermore, since the degree $\mu$ in (1.1) is required $\mu \in(0,1]$, even if $c=0$, the results of the present paper are different from Theorem 1.1.

Acknowledgements

The author would like to thank the handling editors and the anonymous reviewers.

Funding

This work is supported by the Supporting Plan for Excellent Young Talents in Colleges and Universities of Anhui Province (No. gxyq2019166).

Availability of data and materials

Not applicable.

Competing interests

The author declares that they have no competing interests.

Authors' contributions

The author conceived of the study, drafted the manuscript, and approved the final manuscript.

Authors' information

School of Tencent Cloud Big Data, Ma'anshan University, 243100, China. 


\section{Publisher's Note}

Springer Nature remains neutral with regard to jurisdictional claims in published maps and institutional affiliations.

\section{Received: 9 June 2020 Accepted: 7 October 2020 Published online: 16 October 2020}

\section{References}

1. Torres, P.J: Mathematical Models with Singularities-a Zoo of Singular Creatures. Atlantis Press (2015)

2. Plesset, M.S.: The dynamics of cavitation bubbles. J. Appl. Mech. 16, 228-231 (1949)

3. Nagumo, M.: On the periodic solution of an ordinary differential equation of second order. In: Zenkokou Shijou Suudaku Danwakai, pp. 54-61. Springer, Berlin (1944) (in Japanese). English translation in Mitio Nagumo Collected papers, 1993

4. Lei, J., Zhang, M.: Twist property of periodic motion of an atom near a charged wire. Lett. Math. Phys. 60(1), 9-17 (2002)

5. Huang, J., Ruan, S., Song, J.: Bifurcations in a predator-prey system of Leslie type with generalized Holling type functional response. J. Differ. Equ. 257(6), 1721-1751 (2014)

6. Lazer, A., Solimlni, S.: On periodic solutions of nonlinear differential equations with singularities. Proc. Am. Math. Soc. 99, 109-114 (1987)

7. Torres, P.J.: Weak singularities may help periodic solutions to exist. J. Differ. Equ. 232, 277-284 (2007)

8. Hakl, R., Torres, P..:: On periodic solutions of second-order differential equations with attractive-repulsive singularities. J. Differ. Equ. 248, 111-126 (2010)

9. Lu, S., Jia, X.: Existence and uniqueness of homoclinic solution for a Rayleigh equation with a singularity. Qual. Theory Dyn. Syst. 19, 17 (2020). https://doi.org/10.1007/s12346-020-00357-6

10. Lu, S., Xue, R.: Periodic solutions for a singular Liénard equation with indefinite weight. Topol. Methods Nonlinear Anal. 54(1), 203-218 (2019)

11. Chu, J., Torres, P.J., Zhang, M.: Periodic solutions of second order non-autonomous singular dynamical systems. J. Differ. Equ. 239, 196-212 (2007)

12. Cheng, Z., Ren, J.: Multiplicity results of positive solutions foe fourth-order nonlinear differential equation with singularity. Math. Methods Appl. Sci. 38, 5284-5304 (2016)

13. Cheng, Z., Ren, J: Positive solutions for fourth-order singular nonlinear differential equation with variable-coefficient. Math. Methods Appl. Sci. 39, 2251-2274 (2016)

14. Habets, P., Sanchez, L.: Periodic solutions of some Liénard equations with singularities. Proc. Am. Math. Soc. 109, $1135-1144$ (1990)

15. Lu, S., Guo, Y., Chen, L.: Periodic solutions for Liénard equation with an indefinite singularity. Nonlinear Anal., Real World Appl. 45, 542-556 (2019)

16. Cheng, Z., Cui, X., Bi, Z:: Attractive singularity problems for super-linear Liénard equation. Positivity (2019). https://doi.org/10.1007/s11117-018-0615-0

17. Lu, S., Yu, X.: Periodic solutions for second order differential equations with indefinite singularities. Adv. Nonlinear Anal. 9, 994-1007 (2019)

18. Jebelean, P., Mawhin, J.: Periodic solutions of singular nonlinear differential perturbations of the ordinary p-Laplacian. Adv. Nonlinear Stud. 2(3), 299-312 (2002)

19. Zhang, M.: Periodic solutions of Liénard equations with singular forces of repulsive type. J. Math. Anal. Appl. 203(1), 254-269 (1996)

20. Lu, S., Yu, X.: Existence of positive periodic solutions for a neutral Liénard equation with a singularity of repulsive type J. Fixed Point Theory Appl. 21, 31 (2019). https://doi.org/10.1007/s11784-019-0669-z

21. Martins, R.: Existence of periodic solutions for second-order differential equations with singularities and the strong force condition. J. Math. Anal. Appl. 317, 1-13 (2006)

22. Hakl, R., Torres, P.J., Zamora, M.: Periodic solutions of singular second order differential equations: upper and lower functions. Nonlinear Anal. 74, 7078-7093 (2011)

23. Wang, Z: Periodic solutions of Liénard equation with a singularity and a deviating argument. Nonlinear Anal., Real World Appl. 16(1), 227-234 (2014)

24. Kong, F., Liang, Z.: Positive periodic solutions for singular fourth-order differential equations with a deviating argument. Proc. R. Soc. Edinb., Sect. A 148, 605-617 (2018)

25. Kong, F., Lu, S., Liang, Z.: Existence of positive periodic solutions for neutral Liénard differential equations with a singularity. Electron. J. Differ. Equ. 2015, 242 (2015)

26. Gaines, R., Mawhin, J.: Coincidence Degree and Nonlinear Differential Equations. Springer, Berlin (1977)

27. Lu, S., Ge, W.: On the existence of periodic solutions for a kind of second order neutral functional differential equation Appl. Math. Comput. 157, 433-448 (2004) 